\title{
Étude comparative des manifestations cliniques observées chez les patients positifs versus patients négatifs chez les suspects de COVID-19 au CHU Yalgado Ouédraogo de Ouagadougou (Burkina Faso)
}

\section{Comparative study of clinical manifestations observed in positive patients versus negative patients to the suspected COVID-19 at the Yalgado Ouédraogo Teaching Hospital in Ouagadougou (Burkina Faso)}

Savadogo $\mathrm{M}^{1}$, Ouattara $\mathrm{A}^{2}$, Dahani $\mathrm{CK}^{2}$, Nikiéma $\mathrm{O}^{2}$, Traoré $\mathrm{S}^{2}$, Nagréongo $\mathrm{B}^{2}$, Sawadogo $\mathrm{N}^{3}$

1) Service des maladies infectieuses du CHU Yalgado Ouédraogo

2) Service des urgences médicales du CHU Yalgado Ouédraogo

3) Direction de la qualité du CHU Yalgado Ouédraogo

Auteur correspondant : Savadogo Mamoudou Email : $\underline{\text { savadoma@gmail.com }}$

\section{Résumé}

Introduction : La pandémie de COVID-19 en cours n'a pas épargné le Burkina Faso qui a enregistré ses premiers cas depuis le 09 mars 2020. Objectifs : Comparer les manifestations cliniques observées chez les patients positifs au COVID-19 versus patients négatifs à la COVID-19.

Méthodes : Il s'agit d'une étude transversale analytique qui s'est déroulée du 05 avril 2020 au 04 mai 2020. Étaient inclus tous les patients présentant des signes évocateurs de COVID-19 reçus dans la zone d'accueil et de tri des patients suspects du CHU Yalgado Ouédraogo. Les définitions des cas sont celles de l'Organisation Mondiale de la Santé (OMS). La confirmation du diagnostic a été faite au laboratoire grâce à la RT-PCR.

Résultats : En un mois d'activité au niveau de la zone de tri du CHU Yalgado Ouédraogo, 151 cas suspects de COVID-19 ont été reçus. Le test de la COVID-19 a été positif chez 14 cas, soit un taux de positivité de $9 \%$. La majorité des patients (13/14) soit 93\% souffrant de COVID-19 provenaient de la ville de Ouagadougou contre (93/137) soit $68 \%$ des patients négatifs au COVID-19 ( $\mathrm{p}=0,003)$. Sur le plan clinique, une fièvre avec une température supérieure à $38^{\circ} \mathrm{C}$ était retrouvée chez $(4 / 14) 28,5 \%$ des patients COVID+ contre (47/76)62\% des patients COVID- $(\mathrm{p}=0,013)$; une détresse respiratoire était présente chez (4/14) 28\% des patients COVID+ contre $(11 / 122) 9 \%$ des patients COVID- $(\mathrm{p}=0,02)$; quatorze pourcent $(2 / 14)$ des patients COVID+ avaient des algies diffuses contre $2 \%(3 / 128)$ des patients COVID- $(\mathrm{p}=0,04)$; une Spo $<95 \%$ était retrouvée chez $21 \%$ (3/14) des patients COVID+ contre 72\% (36/50) chez les patients COVID- $(\mathrm{p}=0,0005)$.

Conclusion : La COVID-19 devrait être évoquée chez tout patient qui présente des algies diffuses, une détresse respiratoire avec ou sans fièvre.

Mots clés : Comparaison, COVID-19, manifestations cliniques, Ouagadougou, Burkina-Faso.

\author{
Abstract \\ Introduction: The ongoing COVID-19 pandemic has not spared Burkina Faso which has recorded its first cases \\ since March 09, 2020.
}

Objectives : Compare the clinical manifestations observed in COVID-19 positive patients versus COVID-19 negative patients.

Methods: This is an analytical cross-sectional study which took place from April 05, 2020 to May 04, 2020. All patients with signs suggestive of COVID-19 received in the reception and sorting area of suspected patients of the Yalgado Ouédraogo University Hospital were included. Case definitions are those of the World Health Organization (WHO). Confirmation of the diagnosis was made in the laboratory using RT-PCR.

\section{Results}

In one month of activity in the sorting area of the Yalgado Ouédraogo CHU, 151 suspected cases of COVID-19 have been received. The COVID-19 test was positive in 14 cases, a positivity rate of $9 \%$. The majority of patients (13/14) or 93\% suffering from COVID-19 came from the city of Ouagadougou against (93/137) that is $68 \%$ of patients negative for COVID-19 $(\mathrm{p}=0.003)$. Clinically, a fever with a temperature greater than $38^{\circ} \mathrm{C}$ was found in (4/14) $28.5 \%$ of COVID + patients against $(47 / 76) 62 \%$ of COVID- patients $(p=0.013)$; respiratory distress was present in (4/14) $28 \%$ of COVID + patients versus (11/122) $9 \%$ of COVID- patients ( $p=$ $0.02)$; fourteen percent (2/14) of COVID + patients had diffuse pain compared to $2 \%$ (3/128) of COVIDpatients $(\mathrm{p}=0.04)$; Spo $2<95 \%$ was found in $21 \%(3 / 14)$ of COVID + patients against $72 \%$ (36/50) in COVIDpatients $(\mathrm{p}=0.0005)$.

Conclusion: COVID-19 should be considered in any patient who presents with diffuse pain, respiratory distress, with or without fever.

Key words: Comparison, COVID-19, clinical manifestations, Ouagadougou, Burkina-Faso. 


\section{Introduction}

La pandémie de COVID-19 partie de Chine poursuit sa progression à travers le monde malgré les mesures de restriction prises par les Etats [1,2]. Elle a touché à la date du 03 septembre 2020, plus de 26 millions de personnes et tué plus de 863570 décès. Le continent africain compte plus d'un million deux cent mille cas avec 30000 décès à la date du 04 septembre 2020 [3]. Au Burkina Faso à la date du $1^{\text {er }}$ septembre 2020 , le pays comptait 1 386 cas confirmés et 55 décès. Si dans la majorité des cas les manifestations cliniques sont bénignes, l'évolution peut se faire vers un syndrome respiratoire aigu sévère (SRAS) dans certaines circonstances, avec difficultés respiratoires ou essoufflement, imposant une prise en charge dans une unité de réanimation [4]. Si les manifestations de la maladie sont dominées par des signes respiratoires, les connaissances actuelles lui attribuent d'autres manifestations [4,6,7]. En effet la COVID-19 est aujourd'hui considérée comme une maladie pouvant toucher le cœur, les poumons, le tube digestif et le cerveau. Ainsi il a été décrit des accidents vasculaires, des embolies pulmonaires, des troubles du goût et de l'odorat associés à la maladie. Sur le plan étiopathogénique, les nombreuses lésions au niveau des vaisseaux sanguins, occasionnées par le virus, déclencherait une cascade de coagulation qui affecte plusieurs organes expliquant l'atteinte multi-viscérale observée chez certains patients [6]. Plusieurs études avaient rapporté les effets délétères du virus sur les cellules endothéliales, avec comme conséquence des troubles de l'hémostase [3,4,7]. Le polymorphisme clinique de la maladie rend obligatoire le recours aux examens para cliniques pour confirmer son diagnostic. Dans le but de contribuer à la lutte contre la pandémie, l'hôpital Yalgado Ouédraogo de Ouagadougou (Burkina Faso) a mis en place un dispositif de tri des patients suspects de COVID-19. L'objectif de la présente étude était de comparer la symptomatologie observée chez les patients positifs au COVID-19 et ceux des patients COVID-19 négatifs.

\section{Méthodes}

L'étude s'est déroulée à l'hôpital Yalgado Ouédraogo de Ouagadougou (Burkina Faso) durant la pandémie. Il s'est agi d'une étude transversale analytique durant la période allant du 05 avril 2020 au 04 mai 2020. Les patients suspects proviennent aussi bien de la zone de tri que des services d'hospitalisation. A l'admission chaque patient a bénéficié d'une anamnèse et d'un examen physique. L'anamnèse a permis de recueillir les données sociodémographiques des patients (étatcivil, niveau d'études, profession, lieu de résidence...). Ont été inclus les patients présentant une symptomatologie suspecte de COVID-19 telle que définie par l'OMS :
Le cas suspect est défini comme tout patient présentant une infection respiratoire aigüe avec fièvre et au moins un signe respiratoire (toux, difficulté respiratoire), et des antécédents de voyage ou de séjour dans une localité ayant rapporté une transmission communautaire du COVID-19 dans les 14 jrs précédant le début des symptômes; ou tout patient présentant une infection respiratoire aigüe (IRA) avec fièvre et au moins un signe respiratoire (toux, difficulté respiratoire) et ayant été en contact avec un cas confirmé ou probable de COVID-19 dans les 14 jours précédant le début des symptômes; ou tout patient présentant une infection respiratoire aigüe sévère (IRAS) avec fièvre et au moins un signe respiratoire (toux, difficulté respiratoire) nécessitant une hospitalisation et devant l'absence d'une étiologie pouvant expliquer pleinement le tableau clinique.

Le cas probable est un cas suspect pour lequel le test au COVID-19 est indéterminé ou un cas suspect pour lequel le test ne pouvait pas être réalisé pour toute raison, ou les patients présentant des images évocatrices à l'imagerie.

Le cas confirmé est un cas suspect ou probable pour lequel la RT-PCR au COVID-19 est positive.

Pour chaque patient suspect inclus un prélèvement nasopharyngé a été réalisé et acheminé au laboratoire de référence.

N'ont pas été inclus les patients ayant refusé le prélèvement nasopharyngé.

L'analyse des données a été réalisée grâce à un micro-ordinateur avec le logiciel Epi Info dans sa version 7.2.2.2. Le test de Chi-carré a servi à l'analyse statistique. Une valeur de $\mathrm{p}$ inférieure à 0,05 a été considérée comme significative.

Sur le plan éthique : l'anonymat et la confidentialité des informations de chacun des patients ont été respectés dans la collecte et l'analyse des données. Les règles éthiques et déontologiques qui entourent l'exploitation des dossiers cliniques des malades ont été respectées.

\section{Résultats}

En un mois, 151 cas suspects de COVID-19 ont été reçus dont 14 cas confirmés, soit un taux de positivité de $9 \%$. Parmi les patients ayant bénéficié de prélèvement, $20,9 \%$ des patients étaient hospitalisés. Soixante-quatorze virgule huit pourcent des prélèvements ont été réalisés par l'équipe d'investigation de l'hôpital Yalgado Ouédraogo contre $23,8 \%$ par le centre des opérations et de réponse aux urgences sanitaires (CORUS). L'âge moyen des patients était de 60 ans chez les patients COVID positif versus 36,5 ans chez les COVID négatifs. L'âge médian des patients COVID+ était de 64,5 ans avec des extrêmes de 28 ans et 89 ans, contre un âge médian de 57 ans avec des extrêmes d'un mois à 90 ans chez les patients COVID-. Soixante-quatre pourcent des personnes âgées de plus de 60 ans étaient COVID+ contre $36 \%$ des personnes âgées 
de moins de 60 ans $(\mathrm{p}=0,078)$. Les patients âgés de plus de 60 ans avaient 2 fois plus de risque de contracter la maladie que ceux de moins de 60 ans $(\mathrm{OR}=2,28)$. Chez les patients COVID+, le sex ratio $\mathrm{H} / \mathrm{F}$ était de 1 ; tandis que chez les patients COVID-, il était de 1,23. Sur le plan clinique, une fièvre avec une température supérieure à $38^{\circ} \mathrm{C}$ était retrouvée chez (4/14)28,5\% des patients COVID+ contre (47/76) 62\% des patients COVID- $(\mathrm{p}=0,013)$ ; quant à la toux, elle était présente chez (9/14)64\% des patients COVID+ contre (90/129)69\% des patients COVID- $(\mathrm{p}=0,33)$. Une dyspnée était retrouvée chez (8/14) 57\% des patients COVID+ contre (80/32) 60\% des patients COVID- $(p=0,86)$. Une détresse respiratoire était présente chez (4/14) $28 \%$ des patients COVID+ contre (11/122) 9\% des patients COVID- $(\mathrm{p}=0,02)$. Les patients souffrant de détresse respiratoire avaient 4 fois plus de risque de faire une infection au coronavirus $(\mathrm{OR}=4,03)$. Quatorze pourcent (2/14) des patients COVID+ avaient des algies diffuses contre $2 \%$ (3/128) des patients COVID- $(p=0,04)$. Les patients COVID+ avaient 7 fois plus de risque d'avoir des algies diffuses que les patients COVID- $(\mathrm{OR}=6,9)$. Une asthénie était retrouvée chez $28 \%$ (4/14) des patients COVID+ contre 29\% (37/127) chez les patients COVID- $(\mathrm{p}=0,97)$; une douleur thoracique retrouvée chez $7 \%(1 / 14)$ des patients COVID+ contre $12 \%(15 / 125)$ des patients COVID- $(\mathrm{p}=0,5)$. Des céphalées étaient retrouvées chez $21 \%$ (3/14) des patients COVID+ contre $18 \%$ (23/130) chez les patients COVID- $(p=0,35)$. Des vomissements étaient notés chez 14\% (2/14) des patients COVID+ contre 3\% (3/132) chez les COVID-( $p=0,057)$. Les patients COVID+ avaient 5 fois plus de risque $\mathrm{d}$ 'avoir des vomissements $(\mathrm{OR}=5,33)$ et 2 fois plus de risque d'avoir des nausées $(\mathrm{OR}=2,4)$. Un écoulement nasal était retrouvé chez 14\% (2/14) des patients COVID+ contre 6\% (8/128) chez les COVID- $(\mathrm{p}=0,15)$; les patients COVID+ avaient 2 fois plus de risque d'avoir un écoulement nasal $(\mathrm{OR}=2,5)$. Une $\mathrm{Spo} 2<95 \%$ était retrouvée chez $21 \%(3 / 14)$ des patients COVID+ contre $72 \%$ $(36 / 50)$ chez les patients COVID- $(p=0,0005)$. Le tableau I présente les caractéristiques épidémiologiques et cliniques comparées observées chez les patients COVID-19 positifs versus COVID-19 négatifs.
Tableau I : Caractéristiques épidémiologiques et cliniques comparées des patients suspects COVID+ versus COVID-

\begin{tabular}{|c|c|c|c|c|}
\hline Variables & $\begin{array}{c}\text { COVID } \\
+\end{array}$ & $\begin{array}{c}\text { COVID } \\
-\end{array}$ & $\begin{array}{c}\text { Od } \\
\text { ds } \\
\text { Rat } \\
\text { io }\end{array}$ & P-value \\
\hline \multirow[t]{2}{*}{ Sexe } & (M) & $(\mathrm{M})$ & 0,7 & \\
\hline & $50 \%$ & $56 \%$ & 8 & $P=0,36$ \\
\hline $\begin{array}{l}\text { Ages } \geq 60 \\
\text { ans }\end{array}$ & (8)57\% & $36,8 \%$ & $\begin{array}{c}2,2 \\
8 \\
-\end{array}$ & $\mathrm{P}=0,078$ \\
\hline $\begin{array}{l}\text { Provenance } \\
\text { (Ouaga) }\end{array}$ & $\begin{array}{c}(14) 100 \\
\%\end{array}$ & $\begin{array}{c}(79) 68 \\
\%\end{array}$ & & $\begin{array}{c}P \\
=0,003\end{array}$ \\
\hline Fièvre & (12)85\% & (2) $14 \%$ & $\begin{array}{c}1,0 \\
4 \\
0,7\end{array}$ & $\mathrm{P}=0,72$ \\
\hline Toux & (9)64\% & $\begin{array}{c}(90) 69 \\
\%\end{array}$ & $\begin{array}{c}8 \\
0,8\end{array}$ & $\mathrm{P}=0,33$ \\
\hline Dyspnée & (8)57\% & $\begin{array}{c}(80) 60 \\
\%\end{array}$ & $\begin{array}{c}6 \\
0,9\end{array}$ & $\mathrm{P}=0,33$ \\
\hline Asthénie & (4)28\% & $\begin{array}{c}(37) 29 \\
\%\end{array}$ & 7 & $\mathrm{P}=0,49$ \\
\hline $\begin{array}{l}\text { Détresse } \\
\text { respiratoire }\end{array}$ & (4) $28 \%$ & (11)9\% & $\begin{array}{c}4,0 \\
3 \\
1,5\end{array}$ & $\mathrm{P}=0,02$ \\
\hline Mal de gorge & (3) $21 \%$ & $\begin{array}{c}(19) 15 \\
\%\end{array}$ & 6 & $P=0,26$ \\
\hline $\begin{array}{l}\text { Ecoulement } \\
\text { nasal }\end{array}$ & (2)14\% & (8) $6 \%$ & $\begin{array}{l}2,5 \\
1,2\end{array}$ & $\mathrm{P}=0,15$ \\
\hline Céphalées & (3) $21 \%$ & $\begin{array}{c}(23) 18 \\
\%\end{array}$ & 6 & $P=0,36$ \\
\hline $\begin{array}{l}\text { Algies } \\
\text { diffuses }\end{array}$ & (2) $14 \%$ & (3) $2 \%$ & 6,9 & $P=0,04$ \\
\hline Nausées & (2) $14 \%$ & (7) $6 \%$ & $\begin{array}{l}2,4 \\
5,3\end{array}$ & $\begin{array}{c}\mathrm{P}=0,17 \\
\mathrm{P}\end{array}$ \\
\hline Vomissement & (2) $14 \%$ & (4) $3 \%$ & $\begin{array}{c}3 \\
0,1\end{array}$ & $=0,057$ \\
\hline $\begin{array}{l}\text { Saturation en } \\
\mathrm{O}_{2}<95 \%\end{array}$ & (3) $21 \%$ & $\begin{array}{c}(35) 72 \\
\%\end{array}$ & 0 & $\begin{array}{c}\mathrm{P} \\
=0,0005\end{array}$ \\
\hline $\begin{array}{l}\text { Température } \\
>38^{\circ} \mathrm{C}\end{array}$ & (4) $29 \%$ & $\begin{array}{c}(47) 62 \\
\%\end{array}$ & $\begin{array}{c}0,2 \\
4\end{array}$ & $\begin{array}{c}\mathrm{P} \\
=0,013\end{array}$ \\
\hline
\end{tabular}

\section{Discussion}

Notre étude avait pour objectif de comparer la symptomatologie observée chez les patients positifs au COVID-19 et celle des patients COVID-19 négatifs. Dans notre étude le taux de positivité de la COVID-19 était de 9\%. Ce taux était comparable à celui qu'a rapporté Randhawa aux Etats-Unis [7]. Si la maladie semble toucher toutes les couches de la population sans distinction de sexe et d'âge, plusieurs études soutiennent qu'elle serait peu fréquente chez l'enfant. Par contre, les personnes âgées et ceux souffrant de maladies dysimmunisantes seraient plus à risque de faire des 
formes graves $[3,4]$. Les troubles du goût et de l'odorat rapportés dans la littérature, n'ont pas été retrouvés chez nos patients. Cette situation serait due à la petite taille de notre échantillon [12,14]. Dans notre étude, la fièvre, la toux et la dyspnée dominaient la symptomatologie dans les deux groupes (COVID positifs et COVID négatifs) sans lien statistiquement significatif. Plusieurs études avaient établi une chronologie des signes évocateurs de la COVID-19. Pendant que Plaçais, Stanislaw et Esteban soutenaient que la fièvre était le premier symptôme de la COVID-19, Lokken Soutenait que c'était la toux $[10,11,1,13]$. Dans notre étude les troubles digestifs étaient retrouvés chez $9 \%$ des patients contre 33\% dans l'étude américaine [6]. Au cours des infections à coronavirus, la chronologie d'apparition des symptômes digestifs diffère. En effet en cas de COVID-19, les nausées et les vomissements apparaissent avant la diarrhée contrairement au syndrome respiratoire du Moyen-Orient (MERS) et le SRAS-CoV1 où l'inverse est observé.

Conclusion : en comparant la symptomatologie présentée par les patients COVID positifs et celle des patients négatifs au COVID, il est noté beaucoup de similitudes. Il existe cependant un lien entre la détresse respiratoire, les algies diffuses et l'infection à SARS-Cov2 dans notre étude contrairement à la fièvre et la toux. La COVID-19 devrait être évoquée chez tout patient âgé de plus de 60 ans, qui présente des algies diffuses, une détresse respiratoire, des troubles digestifs et un écoulement nasal avec ou sans fièvre.

Conflit d'intérêt : aucun

Références

1) Stanislaw P, Rebecca J, Andrew C M, et al. The 2019-2020 Novel Coronavirus (Severe Acute Respiratory Syndrome Coronavirus. J Glob Infect Dis. 2020; 12(2) : 47-93.

2) Tarek M, Abd El-Aziza, and James D. Stockanda. Recent progress and challenges in drug development against COVID-19 coronavirus (SARS-CoV-2) - an update on the status. Infect Genet Evol. 2020; 83 : 104327.

3) WHO. Clinical management of COVID-19, Interim guidance, 27 May 2020.

4) Jatin M, Jonathan $H$, Ahmed $M$, et al. The Natural History, Pathobiology, and Clinical
Manifestations of SARS-CoV-2 Infections. J Neuroimmune Pharmacol. 2020;21:1-28.

5) Gangqiang G, Lele Y, Kan P, et al. New Insights of Emerging SARS-CoV-2: Epidemiology, Etiology, Clinical Features, Clinical Treatment, and Prevention. Front Cell Dev Biol. 2020 ; 8 : 410.

6) Kaveh $\mathrm{H}$ and al. Gastrointestinal and Hepatic Manifestations of 2019 Novel Coronavirus Disease inaLarge Cohort of Infected Patients From New York : Clinical Implications. Gastroenterology 2020;159(3):1137-40.e2

7) Randhawa K., Fisher H L., Greninger L A, et al. Changes in SARS-CoV-2 Positivity Rate in Outpatients in Seattle and Washington State, March 1-April 16, 2020. JAMA. 2020, 323(22):23342336.

8) Kuldeep D, Sharun K, Ruchi T, et al. Coronavirus Disease 2019-COVID-19. Clin Microbiol Rev. 2020 Oct; 33(4): e00028-20.

9) MS BF. Rapport de situation sur l'épidémie de la maladie à coronavirus (COVID-19) au Burkina Faso. 25 Août 2020.

10) Plaçais L., Richier Q. COVID-19 : caractéristiques cliniques, biologiques et radiologiques chez l'adulte, la femme enceinte et l'enfant. La revue de Médecine Interne volume 41, Issue 5, May 2020, pages 308-318.

11) Lokken EM, Walker CL, Delaney S, Kachikis $\mathrm{N}$ et al. Clinical Characteristics of 46 Pregnant Women with a SARS-CoV-2 Infection in Washington State. 2020 Dec;223(6):911.e1911.e14. doi: 10.1016/j.ajog.2020.05.031.

12) Aziz M. Taste Changes (Dysgeusia) in COVID-19 : A systematic review and metaanalysis. Gastroenterology 2020 Sep;159(3):1132-1133. doi: 10.1053/j.gastro.2020.05.003

13) Esteban OP, Katherine SR, Lenin GB, et al. Clinical, molecular, and epidemiological characterization of the SARS-CoV-2 virus and the Coronavirus Disease 2019 (COVID-19), a comprehensive literature review. Diagn Microbiol Infect Dis. 2020; 98(1) : 115094. Published online $2020 \quad$ May 30. Doi

10.1016/j.diagmicrobio.2020.115094

14) Hossein H, Francesca PS, Hamidreza S, et al. The Global Emergency of Novel Coronavirus (SARS-CoV-2) : An Update of the Current Status and Forecasting. Int J Environ Res Public Health. 2020; 17, 5648 ; doi : 10. 3390/ijerph171656648. 